\title{
COMPORTAMIENTO Y DISEÑO DE MENSULAS DE CONCRETO REFORZADO
}

\section{BEHAVIOR AND DESIGN OF REINFORCED CONCRETE CORBELS}

\author{
PhD. Nelson Afanador García*, PhD. Carlos J. Noriega**, \\ MSc. Gustavo Guerrero $\mathbf{G}^{* *}$ \\ * Universidad Francisco de Paula Santander Ocaña, Facultad de Ingenierías, Programa \\ de Ingeniería Civil, Grupo de investigación en Ingeniería Civil (GIIC). Ocaña, N. STD., \\ Colombia. +57-7-5690088 ext. 222. \\ * * Universidad Francisco de Paula Santander Ocaña, Facultad de Ingenierías, Programa \\ de Ingeniería Mecánica, Grupo de investigación tecnología y desarrollo en Ingeniería \\ (GITYD). Ocaña, N. STD., Colombia. +57-7-5690088 ext. 222. \\ E-mail: \{nafanadorg, cjnoriegas, gguerrerog\}@ufpso.edu.co
}

Resumen: El presente trabajo de investigación realizó un estudio detallado de la formación de las líneas isostáticas en ménsulas de concreto reforzado debido a la imposibilidad de realizar análisis de ellas por la teoría de Euler-Bernoulli dado que no cumple algunas de sus hipótesis. Establecida la geometría del elemento estructural, se realiza su análisis mediante el uso de ambientes de programación como MatLab, Excel, AutoCAD y un programa comercial de modelación en elementos finitos como Ansys con el fin de comparar y calibrar los modelos resultantes de los dos técnicas de análisis. El resultado de esta investigación permitió establecer la familia de isostáticas para un estado plano de tensiones dado que la otra componente de tensiones principales $\left(\sigma_{3}\right)$ es cero para casi todos los puntos de medición y donde no lo fue, su valor es despreciable.

Palabras clave: Ménsula, Líneas Isostáticas, tirante, tensiones, biela.

\begin{abstract}
This research conducted a detailed study of the formation of the isostatic lines in corbel reinforced concrete due to the impossibility of analyzing them by the theory of Euler-Bernoulli as it does not meet some of its assumptions. Once the geometry of the structural element is established, its analysis is carried out by using as programming environments MatLab, Excel, AutoCAD and a finite element commercial modeling program with ANSYS in order to compare and calibrate the models resulting from the two analysis techniques. The result of this investigation established for the family of a plane isostatic stress state since the other component of principal stresses $\left(\sigma_{3}\right)$ is zero for almost all measuring points and where it was not, its value is negligible.
\end{abstract}

Keywords: Corbel, isostatic lines, strut, tension, rod.

\section{INTRODUCCIÓN}

Conocer el comportamiento y diseño de elementos estructurales tipo ménsula es de gran utilidad en las estructuras tales como edificios de uso comercial e industrial, además tiene gran aplicación en edificaciones destinadas a la industria metalúrgica, mecánica y puentes.
La trasmisión de las tensiones de corte en la ménsula de concreto no es nada despreciable y su interacción flexión-cortante la hace más vulnerable a los agrietamientos. Cuando las deformaciones por cortante no son pequeñas relacionadas con las de flexión el uso de la hipótesis de tensión plano propuesta por Bernoulli ya no es aplicable y su comportamiento debe ser estudiado por métodos más precisos. La mayoría de diseñadores utilizan los 
métodos propuestos por el Instituto Americano del Concreto (ACI), el Euro código, entre otros. Este trabajo de investigación se limita al comportamiento y análisis de ménsulas de concreto reforzado, en el cual se establecen las isolíneas de tensiones a través de la programación en Excel, AutoCAD y Ansys v19, su interpretación, tipos de falla (Chakrabarti, Farahani, et al. 1989; Kriz, and Raths, 1965; Mattock, Chen, et al. 1976; Solanki and Sabnis, 1987; Yong and Balaguru 1994; Foster, Powell, et al. 1996) y algunas bases del diseño del elemento estructural, esto le permitirá al lector hacer una comparación entre los diseños establecidos por los métodos ACI, Euro código y el puntal - tensor.

\section{MATERIALES Y METODOS}

Las ménsulas son partes en voladizo que se destinan a resistir la acción de fuerzas concentradas y que por sus dimensiones tiene una distribución de tensiones internas que no pueden ser obtenidos por los métodos tradicionales (Pindula, 2011), la teoría básica de Bernoulli empleada en elementos de concreto, es decir, las secciones de estos elementos no permanece planas durante la flexión y es necesario establecer su comportamiento mediante sus isostáticas.

Las isostáticas son líneas de esfuerzos que permiten comprender los requerimientos de resistencia en cualquier elemento estructural y cada uno de sus puntos son tangentes a las direcciones principales de tensiones, es por ello que son también llamadas trayectorias de esfuerzos principales. En la Figura 1 se muestran las redes isostáticas de compresión y tensión de una ménsula de sección rectangular sometida a flexión.
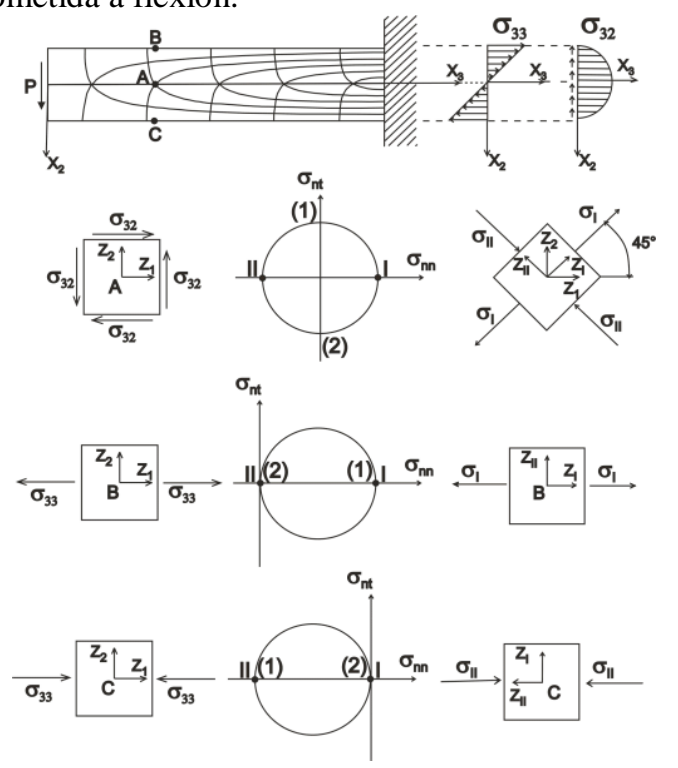

Fig. 1. Isostáticas de una ménsula. (Adaptado de Portela A. y Charafi A., 2002)

Las tensiones tangenciales $\left(\sigma_{32}\right)$ se anulan en las fibras externas superior e inferior y los esfuerzos principales son paralelos a esas fibras, como se muestran en los círculos de Mohr para los puntos B y $\mathrm{C}$, así las tensiones en el punto $\mathrm{B}$ son de tracción $\left(\sigma_{33}\right)$, mientras que las tensiones en el punto $C$ son de compresión $\left(\sigma_{33}\right)$. Es importante anotar que si la pieza es cortada a lo largo de las isostáticas de tracción no habrá deslizamiento debido a que no hay esfuerzos tangenciales y por el contrario se mantienen unidas pues en la dirección perpendicular actúan tensiones principales de compresión (Portela, 2014). (J Plaza, M Núñez, 2017).

Las trayectorias de las tensiones se ven afectados por la influencia de los tensiones cortantes, que van a direccionar la inclinación de las fisuras, las cuales son perpendiculares a las direcciones de los esfuerzos de tracción, es decir la inclinación de las fisuras depende de la inclinación de las tensiones principales de tracción (Dos Santos, 2000); por lo tanto el material del elemento debe estar dispuesto en lo posible a lo largo de las isostáticas (o también llamada resistencia fundamental) verificándose así que estará más adaptada a las solicitaciones a que está expuesta y minimizar la posibilidad de generación de fisuras.

El comportamiento de las ménsulas fue estudiado ampliamente en un programa de pruebas (Kriz y Raths, 1965) y de sus resultados se identificaron los siguientes tipos de falla, ver Figura 2.

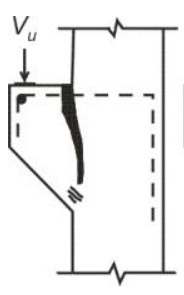

(a)

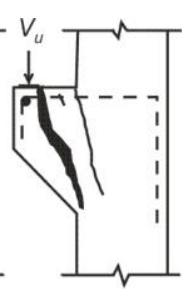

(b)

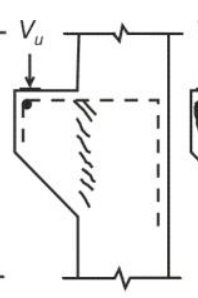

(c)

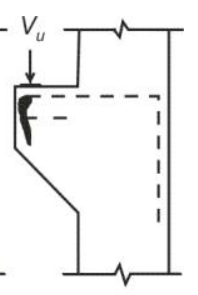

(d)

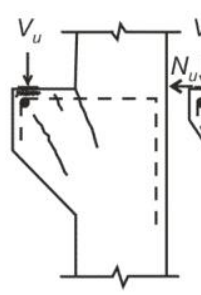

(e)

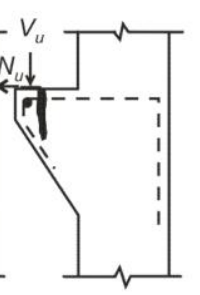

(f)
Fig. 2. Modos de falla en ménsulas. a) Tensión por flexión, b) Tensión diagonal, c) Cortante por 
fricción, d) Fisuración de anclaje, e) Aplastamiento debido a esfuerzos de apoyo y f) Tensión horizontal. Fuente: Park y Paulay, 1975.

La falla de tensión por flexión ocurre cuando se presenta fluencia excesiva del refuerzo a flexión provocando un aplastamiento en el concreto en el extremo inclinado de la ménsula (Fig. 2 a), mientras que la falla por Tensión diagonal se presenta a lo largo del puntal a compresión diagonal después de formarse las grietas por flexión (Fig. 2 b). Una serie de grietas cortas en diagonal se presentan en la unión de la columna y la ménsula, y se denominan falla por cortante de fricción (Fig. 2 c), o si ocurre una fisuración a lo largo del refuerzo a flexión pobremente anclado cuando la carga se aplica cerca al extremo libre se denomina fisuración de anclaje (Fig. 2 d), ocurre una falla por aplastamiento en el apoyo cuando la placa de apoyo es muy pequeña o muy flexible, o cuando la ménsula es demasiado angosta el concreto puede aplastarse en la parte de abajo (Fig. 2 e) y por último se presenta la falla por tensión horizontal cuando se aplica una fuerza horizontal $\mathrm{Nu}$, producto de un efecto dinámico, contracción, flujo plástico o acortamiento por temperatura (Fig. 2 f). Estos tres últimos modos secundarios se presentan con cargas mucho menor a las cuales se pudo haber presentado cualquiera de los cuatro modos principales (Kriz y Raths, 1965).

Existen tres métodos de diseño ampliamente usados en ménsulas, a saber: el código ACI 318 (ACI 318, 2011) en su sección 11.8 (Provisions for brackets and corbels), el Eurocódigo 8 y el Modelo PuntalTensor. Las ménsulas se diseñan para resistir fuerzas de tensión, compresión y cortante de tal forma que no excedan los estados límites.

Las recomendaciones de diseño en ménsulas se originan en el código ACI en 1971 (ACI 318, 1971) con 2 método de diseño: uno basado en relaciones empíricas de Kriz y Raths para ménsulas con a / d $\leq$ 1.0 (donde "a" es la distancia de la carga a la cara del elemento de apoyo y "d" la altura efectiva de la ménsula) y el segundo se basó en la teoría de fricción por cortante con a / d $\leq 0.5$. En 1983 el código fue cambiado y las relaciones empíricas de Kris y Raths fueron omitidas; las recomendaciones de Mattock son las que siguen vigentes hasta ahora (Castillo C. 2007); (R García - León, et al., 2016).

El presente trabajo realizó un estudio del comportamiento y análisis de una ménsula de concreto reforzado de $1.10 \times 1.20 \times 0.40 \mathrm{~m}$ sujeta a una carga concentrada de $1200 \mathrm{kN}$ del cual se conocen las tensiones que se generan en diferentes posiciones de una ménsula, véase algunas tensiones principales en la tabla N.1.

Tabla 1: Tensiones normales y tangente en ménsula

\begin{tabular}{ccccc}
\hline $\mathbf{X}_{\mathbf{1}}(\mathrm{m})$ & $\mathbf{X}_{\mathbf{2}}(\mathrm{m})$ & $\boldsymbol{\sigma}_{\mathbf{1 1}}(\mathrm{MPa})$ & $\boldsymbol{\sigma}_{\mathbf{2 2}}(\mathrm{MPa})$ & $\boldsymbol{\sigma}_{\mathbf{1 2}}(\mathrm{MPa})$ \\
\hline 0.04 & 0.08 & 0.00 & $-2,128.00$ & -0.43 \\
0.16 & 0.88 & 0.46 & $-3,166.00$ & -0.66 \\
0.04 & 1.12 & -0.01 & 0.17 & -0.02 \\
0.16 & 1.12 & -0.64 & $-4,202.00$ & $-1,661.00$ \\
0.04 & 0.48 & -0.01 & $-1,504.00$ & 0.32 \\
0.16 & 0.48 & -0.03 & $-1,522.00$ & 0.83 \\
\hline
\end{tabular}

En la Figura 3 se indica la posición donde se tomó lectura de las tensiones normales $\left(\sigma_{11}\right.$ y $\left.\sigma_{22}\right)$ y la tensión cortante $\left(\sigma_{12}\right)$.

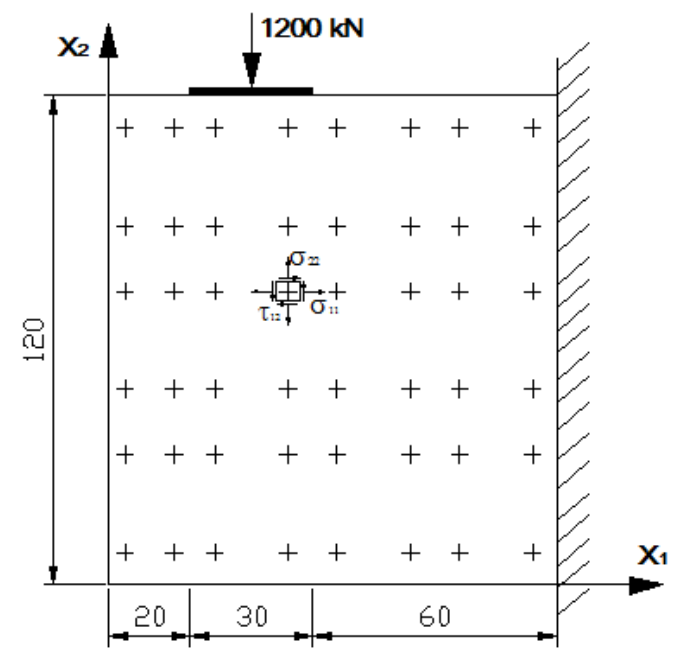

Fig. 3. Ménsula, disposición de sitios de medición de tensiones. (Adaptado de Portela, 2014)

Encontradas las $\sigma_{11}$ y $\sigma_{22}$ y de corte $\left(\sigma_{12}\right.$ o $\left.\tau_{12}\right)$ se establecieron las tensiones principales $\left(\sigma_{\mathrm{I}} \mathrm{y} \sigma_{\mathrm{II}}\right)$ y los ángulos principales ( $\beta_{\mathrm{I}}$ y $\left.\beta_{\mathrm{II}}\right)$, determinados mediante las expresiones de la mecánica de sólidos (ecuación 1 y ecuación 2 ) respectivamente.

$$
\begin{aligned}
& \sigma_{I, I I}=\frac{\sigma_{11}+\sigma_{22}}{2} \pm \sqrt{\left(\frac{\sigma_{11}-\sigma_{22}}{2}\right)^{2}+\tau_{12}^{2}} \\
& \beta_{I}=\frac{1}{2} \tan ^{-1}\left(\frac{2 \tau_{12}}{\sigma_{11}-\sigma_{22}}\right), \quad \beta_{I I}=\beta_{I}+90
\end{aligned}
$$

\section{RESULTADO Y DISCUSIONES}

Las tensiones principales y los ángulos principales fueron calculados en cada punto mediante 
programación en MatLab el cual se indica una muestra de ellos en la tabla 2.

Tabla 2. Tensiones y ángulos principales

\begin{tabular}{cccc}
\hline \multicolumn{3}{c}{ Tensiones Principales } & \multicolumn{3}{c}{ Angulos Principales } \\
$\boldsymbol{\sigma}_{\mathbf{I}}(\mathrm{MPa})$ & $\boldsymbol{\sigma}_{\mathbf{I I}}(\mathrm{MPa})$ & $\boldsymbol{\beta}_{\mathbf{I}}\left(^{\circ}\right)$ & $\boldsymbol{\beta}_{\mathbf{I I}}\left(^{\circ}\right)$ \\
\hline 0.08 & -2.21 & 58.17 & 178.17 \\
0.58 & -3.28 & 52.05 & 172.05 \\
0.17 & -0.01 & 3.95 & 123.95 \\
0.01 & -4.86 & 59.86 & 179.86 \\
0.06 & -1.57 & 58.29 & 178.29 \\
0.35 & -1.89 & 51.75 & 171.75 \\
\hline
\end{tabular}

Mediante la utilización de instrucciones de Excel del tipo insert y concatenar se exportan los resultados en archivo plano para después cambiar a extensión .scr (extensión de archivo de comando). y AutoCAD versión 2014 los lee mediante la instrucción Script, previa definición de las figuras de compresión (vino tinto) y de tracción (verde) mediante bloques para luego con los puntos identificados con " $\mathrm{x}_{1}$ " $\mathrm{y}$ " $\mathrm{x} 2$ ", los esfuerzos principales de tensión (verde) y compresión (vino tinto), además su ángulo de inclinación, se presentan las tensiones en escala 2cm: $10 \mathrm{MPa}$ y geométricas 1:0.1 (ver Figura 4) permitiendo trazar las isolíneas de esfuerzos, además fueron elaboradas en un software comercial de elementos finitos del tipo Ansys ver 19.0 (ver Figura 5),

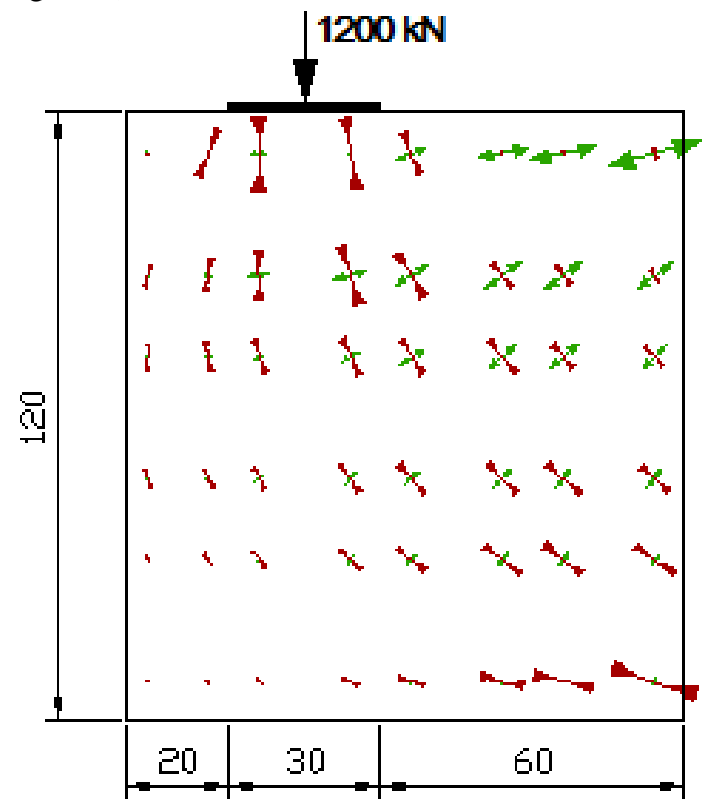

Fig. 4. Esfuerzos y ángulos principales en los sitios de medición.

En la Figura 4, se puede observar que la zona de máximas tensiones de compresión ocurre en el punto de aplicación de la carga y en la parte inferior de la ménsula (zona de unión columna-ménsula inferior) generando un puntal de compresiones inclinado. La zona de máxima tracciones está presente en la parte superior derecha, donde la máxima tensión de tracción es de 9,47 MPa, tensión esta que deberá ser resistida por el refuerzo de tracción dispuesto para tal fin. La parte inferior izquierda esta solicitada a tensiones de compresión muy bajos y por lo tanto esta parte de la ménsula no es necesaria y puede presentarse un ahorro en material hasta la zona donde las tensiones de compresión no excedan la resistencia a compresión del material multiplicada por un factor de mayoración de carga. Es importante el conocimiento de la familia de isostáticas con el fin de establecer la mejor colocación del material resistente de tal manera que no se presenten desperdicios en material o disposiciones erradas en la colocación del material de refuerzo, como puede ocurrir en la ménsula estudiada en la Figura 4 y 5 donde la parte inferior derecha no está siendo solicitada de manera importante pues las tensiones de compresión son del orden de 0,02 MPa y las tensiones de compresión de diseño en forma inconfinada es de $0,85 \mathrm{f}^{\prime} \mathrm{c}$ (puede ser del orden de $17,85,20,83,23,80 \mathrm{MPa}$ entre otros), es decir que las tensiones de compresión llegan a ser hasta del $0,11 \%$ (respecto a $17,85 \mathrm{MPa}$ ) de su máxima capacidad, es por ello que se pudiera utilizar una geometría como la sugerida por Pindula (2.011).

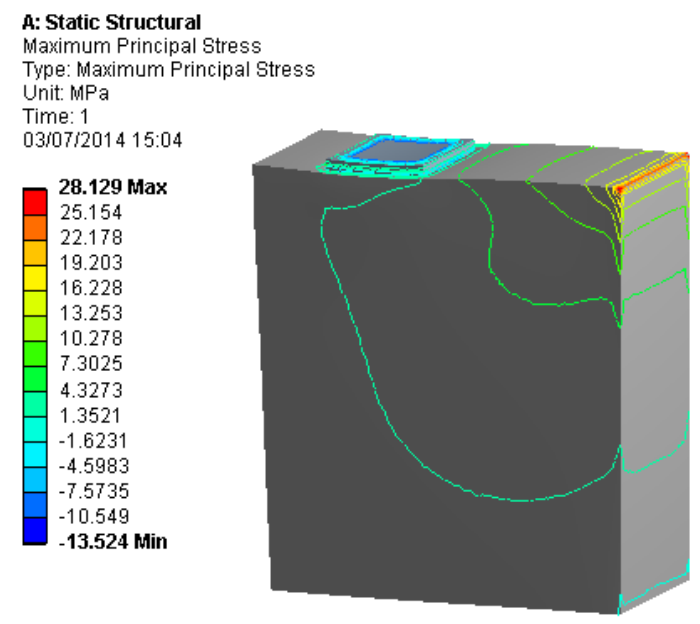

Fig. 5. Isolíneas de tensiones en ménsula de concreto reforzado.

Es visible que hay dos estados de tensiones (estado plano de tensiones) un estado de tensiones de tracciones sensiblemente paralelo a la cara superior de la ménsula y otra de tensiones de compresiones partiendo oblicuamente de la zona cargada hasta la 
parte baja de la unión ménsula y columna; puede así deducirse un esquema mecánico compuesto por una barra traccionada o tirante y una barra comprimida o biela conocido como la teoría de Tirante-Bielas.

Es importante distribuir el acero de refuerzo de tal forma que tenga en cuenta la familia de isostática y evite la formación de fisuras. La armadura que constituye el tirante debe ser distribuida en una altura igual a $0,25 \mathrm{~d}$, donde "d" es la altura útil y su porcentaje no debe ser inferior al 25\% del área bruta en el caso de armaduras de acero A235 y del 15\% del tipo A400 o A500. Además en la zona inferior del tirante debe distribuirse una armadura horizontal suplementaria cuya sección total no sea inferior a 1/4 de la sección de la armadura del tirante (Pindula, 2011). La armadura del tirante debe ser calculada como la relación entre la fuerza resistente en el tirante (calculada) y el valor de la tensión de cedencia de la armadura de refuerzo (depende del material).

\section{CONCLUSIONES}

El comportamiento de las tensiones o familias de isostáticas en los elementos estructuras es fundamental en la comprensión del material y permite establecer la mejor disposición del material resistentes (acero de refuerzo) con fin de optimizar su utilización.

Herramientas como los software de elementos finitos (Ansys, Abaqus, entre otros) permiten establecer la familia de isostáticas, aunque hay que tener cuidado de definir bien los parámetros de entrada. Esto permite ante desconocimiento del comportamiento de un elemento estructural debido a las solicitaciones, establecer las isostáticas de forma de definir bien el refuerzo del elemento.

\section{REFERENCIAS}

American Concrete Institute (ACI) (2011). Comittee 318: Building Code Requirements for Structural Concrete. Farmington Hills, Michigan, American Concrete Institute.

American Concrete Institute (ACI) (1971). Comittee 318: Building Code Requirements for Structural Concrete. Farmington Hills, Michigan, American Concrete Institute.
Castillo M. Carlos A, (2007). Tesis de maestría no publicada "Estudios analíticos y experimentales de ménsulas en extremos de vigas de concreto reforzado" Universidad Nacional Autónoma de México.

Chakrabarti, P. D. Farahani, et al. (1989). "Reinforced and Precompressed Corbels - A Experimental Study." ACI Structural Journal 86(4): pp. 405-412.

Dos Santos B., P., S, (2000). "Comportamento resistente de vigas submetidas à flexão e à força cortante" Apontamentos de Estruturas de concreto I, Universidade de São Paulo.

Foster, S., J., E., Powell, et al. (1996). "Performance of High-Strength Concrete Corbels." ACI Structural Journal 93(5): pp. 1-8.

JEG Plaza, MAR Núñez, (2017) Formación en competencias específicas para la industria del software colombiano. Experiencias del uso del aprendizaje basado en proyectos. Revista Colombiana de Tecnologías de Avanzada, ISSN: 1692-7257

Kriz, L., B., and C. H. Raths, (1965). "Connections in Precast Concrete Structures-Strength of Corbels." Proceedings - Journal of The Presstresed Concrete Institute (1): pp. 16-61.

Mattock, A., K., C., Chen, et al. (1976). "The Behavior of Reinforced Concrete Corbels." Journal of the Prestressed Concrete Institute 21(2): pp. 5277.

Park, R. and T. Paulay, (1975). Reinforced Concrete Structures. New York, Wiley-Interscience.

Portela A., and Charafi A., (2002). "Finite Elements Using Maple. A symbolic programming Approach, Editorial Springer. New York, USA.

Pindula, Jorge, (2011). Análise e dimensionamento de consolas curtas. Verificação da segurança. Apontamentos de Betão Armado II. Universidade Politécnica, Lisboa, Portugal.

R García-León, E Flórez, C Acevedo (2018). Caracterización térmica de mezclas de arcillas utilizadas en la fabricación de productos de mampostería para la construcción. Revista Colombiana de Tecnologías de Avanzada, ISSN: 1692-7257.

Solanki, H. and G. M. Sabnis, (1987). "Reinforced Concrete Corbels-Simplified." ACI Structural Journal 84(5): pp. 428-432.

Yong, Y.-K. and P. Balaguru, (1994). "Behavior of Reinforced High-Strength-Concrete Corbels." ASCE Journal of Structural Engineering 120(4): pp. 1182-1201. 